\title{
INFLUENCIA DEL TIPO DE RIEGO CON AGUA OZONIFICADA EN EL CONTROL DEL NIVEL DE DAÑO DE LA SIGATOKA NEGRA EN BANANO
}

\author{
INFLUENCE OF THE TYPE OF IRRIGATION WITH OZONIZED WATER IN THE \\ CONTROL OF THE DAMAGE LEVEL OF THE BLACK SIGATOKA IN BANANA
}

\author{
ÁNGEL LLERENA HIDALGO', RAFAEL CASTAÑO OLIVA² \\ 1 Universidad Católica de Santiago de Guayaqui l. angel.llerena@cu.ucsg.edu.ec \\ 2 Universidad Católica de Santiago de Guayaquil. oliva_castaño@hotmail.com
}

\begin{abstract}
Se considera a Ecuador el principal productor y exportador de banano en el mundo, lo cual constituye un ingreso importante de divisas al país y una fuente de mano de obra para la población rural directamente e indirectamente. Estudios recientes han demostrado el control efectivo de la enfermedad Sigatoka negra causada por el hongo Mycosphaerella fijiensis con la aplicación de agua ozonizada, a través de un sistema de fumigación con un atomizador a motor. La metodología es aplicable en áreas pequeñas. Sin embargo, en bananeras de grandes extensiones no es aconsejable por el gran gasto de energía. Ante esta situación se propone como alternativa el diseño de un sistema de riego suprafoliar donde la aplicación con agua ozonificada sea distribuida a través del sistema de riego. El objetivo general fue determinar los índices de infección de la enfermedad producida por el hongo Mycosphaerella fijiensis de acuerdo a los diferentes sistemas de riego utilizados, realizando una investigación de campo para diseñar el sistema de riego con diferentes tipos de aspersores. El experimento se realizó en la Hacienda Comargara ubicada en Km 42 de la vía Yaguachi -Jujan, provincia de Guayas, Ecuador. Al evaluar los sistemas de riego con diferentes tipos de aspersores, se determinó que el tratamiento 4 con el tipo de aspersor Xcel Wobbler fue el que presentó los mejores resultados en la evaluación de control de la Sigatoka Negra tanto a nivel de campo como a nivel de laboratorio.
\end{abstract}

PALABRAS CLAVE: 0zono, sistema de riego, sigatoka negra, banano.

\section{ABSTRACT}

Ecuador is considered the main producer and exporter of bananas in the world, which constitutes an important income of foreign currency to the country and a source of labor for the rural population directly and indirectly for the national industry in the production of the derivatives for the Packaging the fruit. Recent studies have been able to demonstrate the effective control of the black Sigatoka disease caused by the fungus Mycosphaerella fijiensis with the application of ozonated water through a fumigation system with a motorized atomizer. This methodology is applicable in small areas however in banana of large extensions is not advisable by the great expense of energy. Given this situation, it is proposed as an alternative the design of a suprafoliar irrigation system where the application with ozonated water is distributed through the irrigation system. The general objective is to determine the infection rates of the disease caused by the fungus Mycosphaerella fijiensis according to the different irrigation systems used, conducting a field investigation to determine the irrigation system with different types of sprinklers. The experiment was performed in the Farm Comargara located at $\mathrm{Km} 42$ of the Yaguachi -Juan road, Guayas province, Ecuador. The effect of the irrigation system with different types of sprinklers was evaluated and it was determined that the treatment 4 with the sprinkler type Xcel Wobbler was the one that presented the best results in the evaluation of black Sigatoka control both at the field level and at the level of laboratory.

KEYWORDS: ozone, irrigation system, black sigatoka, banana. 


\section{INTRODUCCIÓN}

En un trabajo anterior según Llerena, Castaño, y Aguirre (2015) se estudió el efecto de aplicación de ozono disuelto en agua en el control de Sigatoka Negra en el cultivo de banano, en dos zonas agrícolas con condiciones edafoclimáticas diferentes; una en el cantón Baba y otra en el cantón Jujan. Se hicieron evaluaciones en dos períodos climatológicos (invierno y verano) de marzo a noviembre del 2014; combinadas con las prácticas culturales tradicionales de deshoja, deshija, fertilización, y el monitoreo. Para ello se utilizó la escala de signos visuales de 6 puntos de Stover y el método del preaviso temprano. Las variables evaluadas fueron: diámetro del fuste $(\mathrm{D} / \mathrm{F})$, hojas por planta $(\mathrm{H} / \mathrm{P})$, hoja más joven enferma (HMJE) y porcentaje ponderado de infección (PPI) para cada frecuencia de aplicación evaluada. La variable D/F no tuvo diferencia significativa, el número de $\mathrm{H} / \mathrm{P}$ en la mayoría de los tratamientos se inició con un mínimo de 3 hojas hasta un máximo de 14 hojas sanas o partes de ella a cosecha. La HMJE en la mayoría de los tratamientos se inició en la hoja número 3 y el PPI en todos los tratamientos se inició con un mínimo de 0,10 a un máximo de $2,1 \%$. En los testigos los valores para las variables evaluadas fueron de 3 a 9 H/P, HMJE la número 2 y el PPI varió entre 0,3 a 2,6\%. Estos valores del PPI en todos los tratamientos, son equivalentes al grado 2 de la escala de Stover (menores al 5\%). Se utilizó un diseño experimental de bloques completamente al azar (BCA) con cuatro repeticiones. Se estableció la relación existente entre la concentración y frecuencia de aplicación de ozono con el nivel de daño de la Sigatoka Negra. Se utilizaron las comparaciones por contrastes ortogonales para evaluar las frecuencias de aplicación con la dosis utilizada; con lo cual se logró un control total de la infección, cosechándose una fruta de calidad y un promedio de 11 hojas a la cosecha.

En este trabajo se busca evaluar la eficacia del tipo de sistema de riego con agua ozonificada en el manejo del nivel de daño de la Sigatoka Negra en Banano; realizando una investigación de laboratorio y experimental de campo, para establecer un Sistema Óptimo de Riego. Para ello se procedió de la siguiente forma:

1. Determinar la variación de la concentración de ozono en cada uno de los tres tipos de riego bajo estudio, realizando un experimento de campo con mediciones en muestras tomadas a la salida de la boquilla del equipo. Todo ello con la intención de diseñar un cuadro comparativo del nivel de variación de la concentración de ozono.

2. Realizar la siembra a partir de plantas meristemáticas, en parcelas experimentales y de control, para lograr plantas uniformes; midiendo altura de planta, diámetro del fuste y emisión foliar.

3. Comparar cada sistema de riego respecto a su eficacia en el control del nivel de daño, realizando aplicaciones cada 4-8 días en todas las parcelas y evaluando el nivel de daño para identificar el tipo de riego óptimo.

4. Determinar los niveles de fertilidad del suelo y de la planta de acuerdo con los sistemas de riego utilizados; realizando análisis de laboratorio para elaborar una tabla de análisis que permita el diseño de un Programa de Fertilización.

MANEJO DE LA SIGATOKA NEGRA (MYCOSPHAERELLA FIJIENSIS)

La enfermedad se ha dispersado por todo el continente americano y el Caribe. Las zonas más afectadas por la Sigatoka se caracterizan por tener precipitaciones mayores a $1.400 \mathrm{~mm}$ anuales, humedad relativa mayor a $80 \%$ y temperatura promedio entre 23 a $28{ }^{\circ} \mathrm{C}$ (Álvarez, Pantoja, Gañan, y Ceballos, 2013). En los diferentes países han ocurrido severas epidemias, que han obligado a intensificar las medidas de combate y han puesto de manifiesto la necesidad de estrategias de manejo integrado de la enfermedad para mitigar su impacto negativo (Guzmán y Villalta, 2006).

\section{BIOLOGÍA DE LA SIGATOKA NEGRA}

$M$. füiensis es una destructiva enfermedad foliar (Guzmán y Paladines, 2015) que se reproduce en forma asexual y sexual. La reproducción asexual se presenta en lesiones jóvenes de la enfermedad (estrías 2 y 3 y el primer estadio de mancha). La fase sexual, de mayor importancia en el desarrollo de la enfermedad, se produce en las lesiones maduras en estructuras denominadas pseudotecios; en cuyo interior se encuentran las ascosporas, las cuales son liberadas al ambiente en períodos de alta humedad para ser dispersadas hasta largas distancias por las corrientes de aire (Stover, 1986) (Martínez, Villalta, Soto, Murillo y Guzmán, 2011).

Actualmente el control de la Sigatoka Negra se centra en la aplicación de fungicidas quími- 
cos. Sin embargo, el patógeno ha desarrollado resistencia aumentando los costos de control y el impacto negativo sobre el ambiente; uniéndose ello a la exigencia de los consumidores por una fruta cada vez más libre del uso de plaguicidas.

El riego con agua ozonizada desinfecta las raíces y el sustrato (Panorama-agro, 2015), proporciona oxígeno a la raíz liberándola de virus, bacterias y hongos (Oz-air, 2012). En concentraciones menores consiste en irradiar aire con luz ultravioleta (Tecnozono, 2015). El ozono también es producido por los rayos ultravioleta (UV) del sol (Lenntech, 2016). El uso de esta metodología en el sector agrícola aparece como una buena alternativa para eliminar residuos tóxicos (CosemarOzono, 2016). Además, el ozono se descompone en oxígeno (AspOzono, 2015), por lo que no resulta peligroso y mucho menos contaminante.

METODOLOGÍA

El experimento se realizó en la Hacienda Comargara (Tabla 1) ubicada en el cantón $\mathrm{Ba}^{-}$ querizo Moreno (Jujan), provincia del Guayas, Ecuador.

TABLA 1. CARACTERÍSTICAS DE LA HACIENDA COMARGARA

\begin{tabular}{l|llr}
\hline LONGITUD OESTE & $790^{\circ} 35^{\prime} 28$ & $\begin{array}{l}\text { Precipitación } \\
\text { anual }\end{array}$ & 1369.5 \\
\hline LATITUD SUR & $10^{0} 57^{\prime} 30 \mathrm{~S}$ & $\begin{array}{l}\text { Temperatura } \\
\text { media anual }\end{array}$ & 25.59 \\
ALTITUD & $9 \mathrm{msnm}$ & $\begin{array}{l}\text { Humedad media } \\
\text { relativa anual }\end{array}$ & 79.33 \\
SUELO & Franco arcilloso & $\mathrm{pH}$ & 6.2 \\
\hline
\end{tabular}

El trabajo consistió en la aplicación de ozono disuelto en agua y aplicado al follaje de las plantas de banano, a través de diferentes tipos de aspersores del sistema de riego y fumigación. La aplicación del agua con ozono en todos los tratamientos en estudio se realizó con intervalos de cuatro días en la época lluviosa y cada ocho días en la época seca. Estas aplicaciones se realizaron con la finalidad de controlar el efecto nocivo del hongo Mycosphaerella en la hoja del banano y comparar la eficiencia de los diferentes sistemas de riego de agua con ozono para el manejo de Sigatoka Negra.

Se construyó un generador de ozono por corona de descarga, compuesto por un transformador de 110 Voltios AC a 6000 Voltios DC. Este transformador envía la corriente a un generador con material dieléctrico, donde se produce el ozono; el cual es alimentado por una fuente de oxígeno al $95 \%$, y a su vez inyectado mediante un Venturi al agua del sistema de riego que se aplica en la plantación. En las fumigaciones, las aplicaciones se realizaron mediante máquinas nebulizadoras a motor con una periodicidad de cuatro y ocho días.

El diseño estadístico fue de bloques completamente al azar con cinco tratamientos que corresponden a los diferentes tipos de aspersores y métodos de aplicación del ozono. El ensayo tuvo 4 repeticiones con Andeva.

MÉTODO

Se empleó un método experimental de campo. Las plantas fueron cultivadas a nivel de campo, para lo cual se procedió a realizar el trasplante de plántulas obtenidas a través de meristemas con una distancia de siembra de $2.40 \times 2.60$ a tres bolillos.

\section{DISEÑO DE LA INVESTIGACIÓN}

Se analizaron las siguientes variables no controlables: clima, tipo de suelo, el manejo que el productor realiza como fertilización, podas sanitarias, etc. Por lo que se optó por realizar una selección de plantas que cumplieran con las siguientes características:

- Una altura promedio entre $1.5 \mathrm{~m}$. y $2.0 \mathrm{~m}$ con un mínimo de 9 hojas.

- Las plantas utilizadas en cada uno de los ensayos fueron distribuidas en una área de 0.5 ha.

- Las plantas fueron marcadas y numeradas de tal manera que nadie las pudiera tocar durante el proceso de evaluación.

- Se evaluó el desarrollo de la enfermedad y se determinó el índice de severidad.

\section{TÉCNICAS DE RECOGIDA DE DATOS}

La recolección de datos se realizó en los campos de Marcin I y de Limoncito, colectando hojas afectadas con Sigatoka. Para la identificación de la infección se efectuaron observaciones en el estéreomicroscopio y en el microscopio con cámara. Se prepararon placas portaobjetos con las esporas seleccionadas, las cuales se identificaron y guardaron debidamente. Asimismo, se conformó un archivo fotográfico.

Para evaluar la toxicidad se extrajeron hojas de plantas de banano. El número de esporas en suelo y sustrato se determinó a través del conteo directo de acuerdo al método de Stover (1986) (Jiménez, 2008). 


\author{
Datos evaluados \\ Datos Agronómicos - cada quince días \\ - Altura de plantas \\ - Diámetro del pseudotallo \\ - Número de hojas totales \\ - Número de hojas presentes por semana \\ Datos sanitarios \\ - Severidad de la enfermedad de acuerdo a la escala de Stover. \\ Datos de producción \\ - Días a la cosecha \\ - Peso de racimo total \\ - Número de manos \\ TRATAMIENTOS \\ T1 Fumigación bomba nebulizadora cuatro y ocho días \\ T2 Método de riego aéreo con aspersor Smooth drive cuatro y ocho días \\ T3 Método de riego aéreo con aspersor M. Wobbler cuatro y ocho días \\ T4 Método de riego aéreo con aspersor Xcel Wobbler cuatro y ocho días \\ T5 Testigo convencional \\ Técnicas y modelos de análisis de datos \\ Para aquellos parámetros en que fue necesario realizar comparaciones entre \\ resultados de tratamientos, se efectuó un análisis de varianza de un \\ factor con el $0.05 \%$ de probabilidad.
}

RESULTADOS DIÁMETRO DE FUSTE

Se observó un mayor D/F de las plantas que provenían de meristemas. Los sistemas de riego y los programas de fertilización fueron los mismos en ambos ensayos. Estos concuerdan con los resultados obtenidos por Rodríguez-Gaviria y Cayón (2008) según se muestra en la Figura 1.

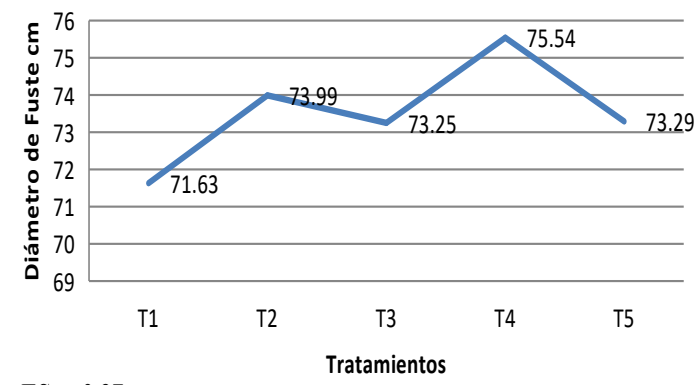

$\mathrm{ESx}=0,97$

Figura 1. Diámetro de Fuste a los 90 dds (cm). Ensayo Jujan $(p<=0,97)$

NÚMERO DE HOJAS TOTALES

Los efectos benéficos de la metodología del ozono mezclado con agua no solo se manifiestan en un control efectivo del hongo Mycosphaerella, sino también que ayuda a los procesos fisiológicos de la planta, tanto a la fotosíntesis como a la respiración al tener una mayor disponibilidad de oxigeno libre. Ello se ve reflejado en un mayor número de hojas en la planta de banano. Esto se pone de manifiesto en la Figura 2 donde el tratamiento 4 presenta el mayor número de hojas con respecto a los otros tratamientos.

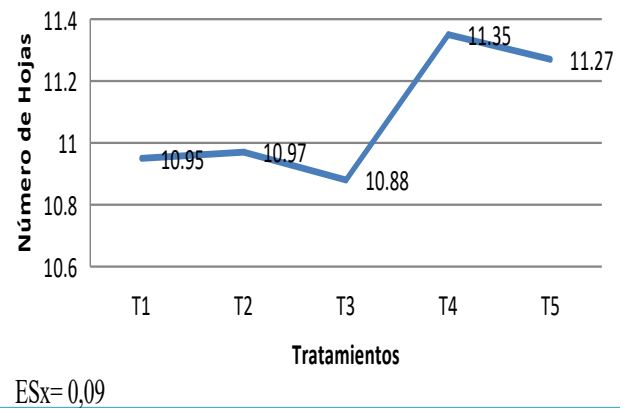

Figura 2. Número de hojas a los 90 dds. Ensayo Jujan significativas $(p<=0,09)$

Es necesario considerar que los factores climáticos presentes durante el período de evaluación, así como la influencia de barreras rompevientos naturales existentes, pudieron tener alguna incidencia en la disminución de la enfermedad.

\section{DATOS DEL ENSAYO}

Después de haber realizado el análisis de la infección en los tratamientos en estudio se observa una disminución en los niveles durante las semanas 17, 18, 19 y 20. Al evaluar la hoja libre de estría (h+vle), ocupa el primer lugar con mayor cantidad de hojas 10.8 y h+vle 9.7 las parcelas que corresponde al tratamiento 4 que representa xcel -Wobbler. El segundo lugar corresponde al tratamiento 1 que representa la aplicación con aspersión Xcel-wobbler invertido con un total de 10.6 hojas y la h+vle 8.1 con el nivel más alto. En tercer lugar el tratamiento 3 donde se utiliza la aspersión del agua ozonizada a través del $M$. Wobbler $U$ con un número de hojas de 10.2 en los niveles más bajos y h+vle 8.1 en los niveles más altos. En cuarto lugar el tratamiento 2 en el cual se evalúa el aspersor Smooth Drive normal con una cantidad de hojas de 9 en los niveles más bajos y h+vle de 8.1 en los niveles altos de infección. En el último lugar se encuentra el tratamiento 5 que corresponde al testigo que presenta una cantidad de hojas equivalente a 9.3 y la h+vle en los niveles más alto de infección 9.2 (Tabla 2).

Luego de soportar las condiciones adversas debido al cambio climático donde se produjeron precipitaciones mayores a las normales, se pudo comprobar la eficiencia de la metodología del uso de ozono al comprobar los resultados de la cosecha con plantas que tenía un promedio de 17 y 18 hojas libres de estrías con un peso promedio de $46 \mathrm{Kg}$ con un número de manos igual a 10. Lo que representa 26 clúster aproximadamente con un ratio de 1.6 constituyendo 
TABLA 2. RESULTADOS DEL USO DE AGUA OZONIZADA PARA EL CONTROL DE LA SIGATOKA NEGRA EN BANANO. ENSAYO EN JUJAN

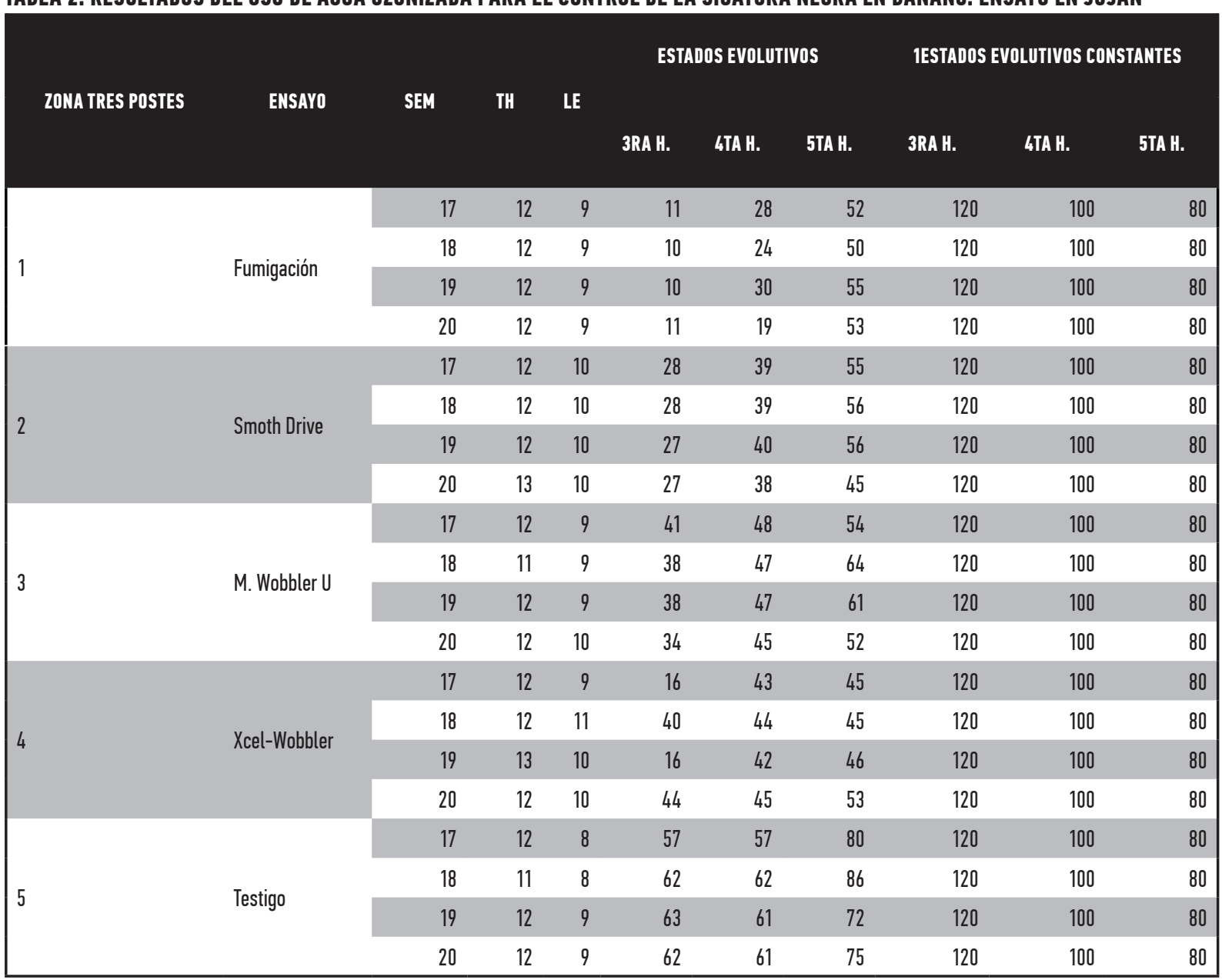

un excelente rendimiento. Se considera que el avance es muy rápido pues la hoja más joven enferma es, en todos los tratamientos, la número 3. El grado ponderado de infección es relativamente bajo y puede ser controlado o manejado utilizando el agua ozonificada; pues no existen diferencias significativas entre las frecuencias de aplicación evaluadas en este estudio según se muestra en la Tabla 3. Estos resultados se complementan con los obtenidos por Hidritec (2011) donde recomienda el uso de $4 \mathrm{ml}$ de ozono /ha sin efectos fitotóxicos sobre la planta y un buen control de la Sigatoka Negra.

\section{DATOS DE PRODUCCIÓN: PESO DE RACIMO}

Los promedios obtenidos en el peso de los racimos de los diferentes tratamientos no presentaron diferencias estadísticas entre el testigo y los tratamientos 1, 3 y 4 (Figura 3). Debido a que probablemente la influencia de ozono sobre el suelo también resulta un elemento con un poder oxidante de los nutrientes del suelo que se encuentran unidos a las micelas coloidales de este; provocando su disponibilidad para las plantas. Esto concuerda con lo manifestado por Ramírez y Sainz (2012) donde indican que el ozono posee un poder oxigenante mayor que el del oxígeno normal y por ello mejora el proceso respiratorio a nivel celular.

TABLA 3. RESULTADOS DEL ANÁLISIS DE LOS TRATAMIENTOS

\begin{tabular}{|llrr|}
\multicolumn{1}{c}{ TRAT. } & \multicolumn{1}{c}{ PARCELAS } & TH & LE \\
\hline T1 & Fumigación & 10,6 & 8,1 \\
T2 & Smoth Drive & 9 & 8,1 \\
T3 & M. Wobbler U & 10,2 & 8,1 \\
T4 & Xcel-Wobbler & 10,8 & 9,7 \\
T5 & Testigo & 9,3 & 8,6 \\
\hline
\end{tabular}

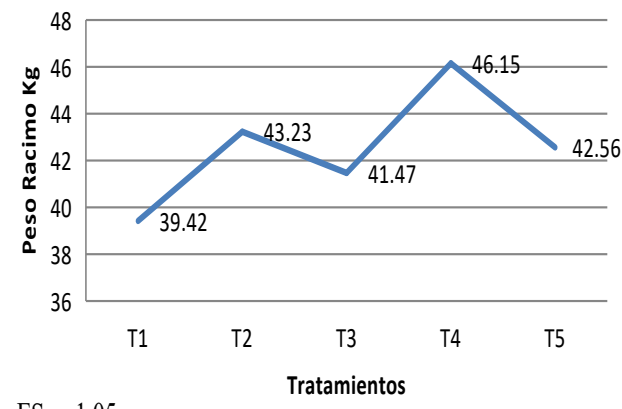

$\mathrm{ESx}=1,05$

Figura 3. Peso de racimos $(\mathrm{kg})$ a la cosecha. Ensayo Jujan $(p<=1,05)$ 


\section{NÚMERO DE MANOS}

En lo que respecta al número de manos los resultados siguieron el mismo comportamiento de no presentar diferencias estadísticas. Sin embargo, se encontraron diferencias numéricas; donde los promedios de Jujan presentaron un mayor número de manos según se muestra en la Figura 4.

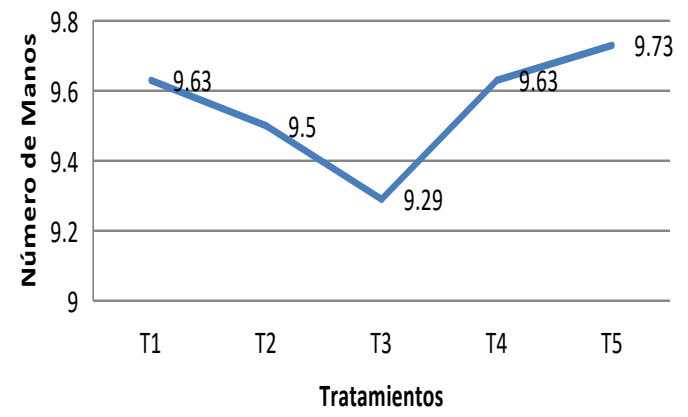

$\mathrm{ESx}=0,12$

Figura 4. Número de manos por racimo a la cosecha. Ensayo Jujan $(p<=0,12)$

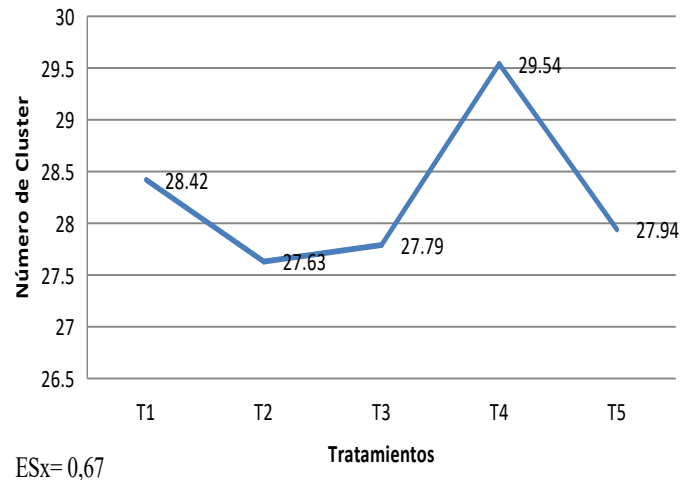

Figura 6. Evaluación de la incidencia de Sigatoka Negra en número de clúster. Ensayo Jujan.

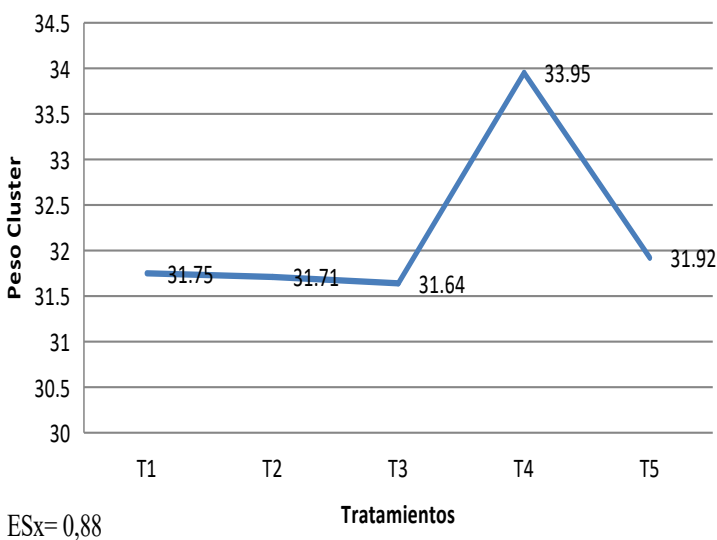

Figura 7. Evaluación de la incidencia de Sigatoka Negra en peso clúster. Ensayo Jujan
NÚMERO DE CLÚSTER

En las Figuras 6 y 7 se puede evidenciar el número de clúster que se obtienen de un racimo de banano cuando la planta es tratada con ozono; ya que además de controlar la infección causada por la Sigatoka Negra, estimula los procesos de solubilidad de los nutrientes del suelo. Ello se expresa en un mayor número y en un mayor peso de clúster, incrementándose el ratio (raci$\mathrm{mo} /$ caja).

\section{DISCUSIÓN DE RESULTADOS}

Los resultados obtenidos en esta investigación constituyen un punto de partida para la disminución gradual del uso de fungicidas químicos para el control de la Sigatoka Negra. Estos resultados coinciden también con trabajos realizados en Cuba, Japón y Nueva Zelanda. Cherry (2016) manifiesta que un planteamiento sencillo para mantener los cultivos limpios se logra con fumigación y los lavados con ozono; los cuales están teniendo un uso cada vez mayor en la industria agrícola. El agua ozonizada utilizada para el riego es otra forma de aplicación del ozono para eliminar los microorganismos que pongan en peligro los cultivos (Ferpa-graphic, 2011). La innovadora compañía neozelandesa Biofume Limited está tomando la iniciativa hacia opciones baratas y eficaces enfocadas al ozono; lo cual concuerda con los objetivos de nuestra investigación

Una alternativa importante que se ha logrado identificar con los resultados de este proyecto es el uso eficiente del ozono para hacer explotar las moléculas de las paredes celulares, de modo que los organismos no pueden desarrollar una resistencia hacia él. Esto supone una ventaja enorme sobre los fumigadores químicos. Las lesiones causadas por la Sigatoka Negra son más abundantes y notorias en el envés de la hoja (Zhiñin, 2016), causando pérdidas en la calidad de la fruta, que reduce su producción de exportación (Calle y Yangali, 2014).

La Universidad Católica de Santiago de Cuayaquil ha presentado recientemente un sistema móvil patentado de fumigación de ozono que puede encajarse en el equipo existente y usarse de forma eficaz para el control de plagas y hongos, sin necesidad de productos químicos perjudiciales. Este sistema ha permitido aumentar el tiempo de aplicación de ozono; observándose además, que el suelo se acidifica y el fósforo asimilable aumenta (Sinc, 2011).

Un aspecto importante del uso de esta metodología lo constituye la disminución del uso de 
fungicidas químicos causante de la contaminación ambiental y de la pérdida de biodiversidad. Permite también que los empleados del sector agrícola puedan trabajar de modo más saludable y con menos riesgos (Innovagri, 2015).

\section{CONCLUSIONES}

1. El mayor grado de infección se presentó en el tratamiento 2 que corresponde a Smoth Drive, con un número total de hojas correspondiente a nueve y hojas libres de estrías de 8.1 de acuerdo a la metodología de Stover.

2. En lo que respecta a los niveles de fertilidad obtenidos del promedio del análisis de suelo, el tratamiento cuatro presenta los valores más altos en concentraciones de 28 ug / ml. Lo que representa una diferencia significativa con el testigo que presentó una concentración de 11ug / ml para la concentración de Nitrógeno. La misma tendencia se manifestó en los demás elementos testados.

Los niveles de fertilidad como resultado del análisis foliar no presentan diferencias significativas entre los tratamientos (3.27 \%) donde se aplicó el ozono. Sin embargo, en tratamiento testigo presentó los valores más bajos $(3.1 \% \mathrm{~N})$.

3. Se logró determinar la acción específica del ozono sobre el micelio de Mycosphaerella fijiensis al observar la detención del crecimiento del micelio $(2.75 \mathrm{~cm})$ en comparación con el tratamiento testigo donde no se aplicó ozono. El micelio creció a valores de $4.75 \mathrm{~cm}$ comprobándose la diferencia estadística altamente significativa con la concentración utilizada en el ensayo.

4. El diseño del sistema óptimo de riego se fundamenta en el tratamiento 4 con aspersor Xcel - Wobbler; en el cual se obtuvo el mejor control y manejo de la Sigatoka Negra, el mayor peso del racimo, número de clúster, número de manos, número de hojas y un mayor $\mathrm{D} / \mathrm{F}$.

\section{REFERENCIAS BIBLIOGRÁFICAS}

Álvarez, E., Pantoja, A., Gañán, L. y Ceballos, G. (2013). Estado del arte y opciones de manejo del Moko y la Sigatoka Negra en América Latina y el Caribe. Cali, Colombia: CIAT - FAO.

AspOzono. (2015). Ozono para la Agricultura (invernaderos, cultivos hidropónicos, agricultura ecológica). Recuperado de http://www. aspOzono.es/invernaderos-agricultura.asp
Calle, H. y Yangali, J. (2014). La Sigatoka Negra en el Ecuador. En La Sigatoka Negra en el Ecuador. Primer Seminario Internacional Metodología para la Evaluación de Prueba de Eficacia para Plaguicidas en los principales cultivos del Ecuador. Recuperado de http://www.agrocalidad.gob.ec/wp content/uploads/2014/12/ PresentacionSigatokaH-Calle-JYangali.pdf

CosemarOzono. (2016). Prevención y control de Plagas y enfermedades en suelos agrícolas con Ozono. Recuperado de http://www.cosemarOzono.es/blog/2016/08/plagas-enfermedadessuelos-agricolas/

Cherry, R. (2016). Un planteamiento sencillo para mantener los cultivos limpios. Recuperado de http://www.freshplaza.es/article/96470/ Un-planteamiento-sencillo-para-mantenerlos-cultivos-limpios

Ferpa graphic. (2011). Aplicaciones del ozono en la agricultura. Recuperado de http://www. ferpa-graphic.com/index.php/productos/ sistemas-deozono/agricultura-y-ganaderia/ agricultura-y-ozono

Guzmán, M. y Paladines, P. (2015). Sigatoka Negra. Croplife. Recuperado de http://www. croplifela.org/es/plaga-del-mes?id=163

Guzmán, M y Villalta, R. (2006). Efecto del método de control de malezas sobre el desarrollo de la Sigatoka negra y la producción del banano (Musa AAA, cv. Grande naine). Corbana 33(60), 50-52.

Hidritec. (2011). El Ozono en la agricultura. Recuperado de http://www.hidritec.com/hidritec.

Innovagri. (2015). El uso de Ozono en la agricultura para incrementar los rendimientos de más de 250 cultivos. Recuperado de http://www. innovagri.es/actualidad/asepsia.html

Jiménez, M. (2008). Effect of the nutritional status of banana (Musa sp.) on leaf disease infestation by Mycosphaerella fijiensis Morelet in Ecuador. Universidad Católica de Leuven. Bélgica. Faculteit Bio-ingenieurswetenschappen KU Leuven.

Lenntech. (2016). Qué es el Ozono. Recuperado de http://www.lenntech.es/biblioteca/Ozono/ preguntas-mas-frecuentes/faqOzono-esp.htm

Llerena, A., Castaño, R. y Aguirre, C. (2015). Relación de la concentración y frecuencia de aplicación de ozono con el nivel de daño de la Sigatoka Negra en banano. Diseño de un protocolo de riego con agua ozonificada. Alternativas, 16 (2), 66-75.

Martínez, I., Villalta, R., Soto, E., Murillo, G., y Guzmán, M. (2011). Proyecto demostrativo con implementacion de BPA en el cultivo del bana- 
no. Corporación Bananera Nacional, Dirección de Investigaciones Seccion de Fitopatologia. Costa Rica: CORBANA. Recuperado de http:// www.infoagro.net/programas/ambiente/ pages/adaptacion/ casos/Sigatoka.pdf

Oz-air. (2012). Ozone in agriculture | ozone water treatment Machine suppliers | Agricultural Water Systems Manufacturers | India. Recuperado de http://www.oz-air.com/agriculture. html

Ramírez, J. y Sáinz, R. (2012). El Ozono en la agricultura y el bienestar. México. DF: Universidad Autónoma de Sinaloa.

Rodríguez-Gaviria, A. y Cayón, G. (2008). Efecto de Mycosphaerella fijiensis sobre la fisiología de la hoja de banano. Agronomía Colombiana 26(2), 256-265.
Sinc. (2011). El Ozono es efectivo para desinfectar suelos agrícolas. Recuperado de http://www. agenciasinc.es/Noticias/El-Ozono-es-efectivopara-desinfectar-suelos-agricolas

Stover, R.H. (1986). Disease management strategies and the survival of the banana industry. Ann. Rev. Phytopathol. 24, 83-91.

Tecnozono. (2015). Ozono. Portal de medio ambiente. Recuperado de http://www.tecnOzono. com/Ozono.htm

Zhiñin, I. A. (2016). Evaluación del fungicida orgánico pk 50 en Sigatoka Negra mycosphaerella fijiensis $\mathrm{m}$ bajo condiciones de campo y laboratorio. Trabajo de titulación. UTMACH, Unidad Académica de Ciencias Agropecuarias, Machala, Ecuador. 